\title{
Probing planet nurseries with rare isotopologues of $\mathrm{CO}$
}

\author{
Sarah E. Dodson-Robinson ${ }^{1}, \mathrm{Mo} \mathrm{Yu}^{1}$ and Karen Willacy ${ }^{2}$ \\ ${ }^{1}$ Astronomy Department, University of Texas, \\ 2515 Speedway Dr C1400, Austin, TX 78712, USA \\ ${ }^{2}$ Jet Propulsion Laboratory, California Institute of Technology, \\ 4800 Oak Grove Drive, Pasadena, CA 91109, USA
}

\begin{abstract}
The properties of planet-forming midplanes of protostellar disks remain largely unprobed by observations due to the high optical depths of common molecular lines. However, rotational emission lines from rare isotopologues may have optical depth near unity in the vertical direction, so that the lines are strong enough to be detected, yet remain transparent enough to trace the disk midplane. We have computed chemical models of protostellar disks including different $\mathrm{C}$ and $\mathrm{O}$ isotopes and detailed photochemical reactions. The $\mathrm{CO}$ condensation front is in the giant planet-forming region, within $20 \mathrm{AU}$ of the star. We show that the optical depths of low-order rotational lines of $\mathrm{C}^{17} \mathrm{O}$ are around unity, which suggests that it may be possible to see into the disk midplane using $\mathrm{C}^{17} \mathrm{O}$. In lower-mass disks, the slightly more abundant $\mathrm{C}^{18} \mathrm{O}$ is a possible midplane probe. ALMA observations would provide estimates of the disk midplane temperature if $\mathrm{CO}$ ice line were spatially resolved. With our computed $\mathrm{C}^{17} \mathrm{O} / \mathrm{H}_{2}$ abundance ratio, we would also be able to measure disk surface densities from the fluxes of low-order $\mathrm{C}^{17} \mathrm{O}$ transitions.
\end{abstract}

\section{Introduction and Methods}

The temperature, density and composition of proto-planetary disks determine materials available for planet formation. However fundamental properties of protostellar disks such as midplane temperature, turbulent visosity, and disk mass are currently poorly constrained by observations. Here we seek a molecule that can be used to probe planetforming protostellar disk midplanes, as opposed to disk surfaces. We focus here on CO isotopologues - molecules with a rare isotope in place of one of the atoms - because their transitions are readily observable with ALMA and they are extremely volatile, which places their condensation fronts as far from the star as possible to facilitate angular resolution of the $\mathrm{CO}$ distribution in nearby disks

We have computed chemical models of evolving disks that include $\mathrm{C}$ and $\mathrm{O}$ isotopes. The evolving disk models from Landry et al. (2013) provide the temperature and density used as input to the chemical model. The computational method is similar to that of Dodson-Robinson et al. (2009). In the Landry et al. (2013) models, the heating is provided by stellar radiation and the magnetorotational instability, which gives a hot disk surface and a cold midplane. After we have computed the abundances of each CO isotopologue as a function of time, we calculate the optical depth of each isotopologue as a function of distance from the star by integrating the absorption efficiency over the vertical disk height. 


\section{Results and Summary}

Figure 1 shows time snapshots of the optical depths in the $\mathrm{J}=3 \rightarrow 2$ transition of the $\mathrm{CO}$ isotopologues as a function of distance from the star. We find that the rare isotopologues of $\mathrm{CO}$ are good probes of the planet-forming midplane of the disk. A given tracer works best when the optical depth is near unity, as observations can peer into the disk midplane while maximizing the observed signal. $\mathrm{C}^{17} \mathrm{O}$ is most effective as a probe of the inner $30 \mathrm{AU}$ of the disk while $\mathrm{C}^{18} \mathrm{O}$ probes the outer regions of the disk.
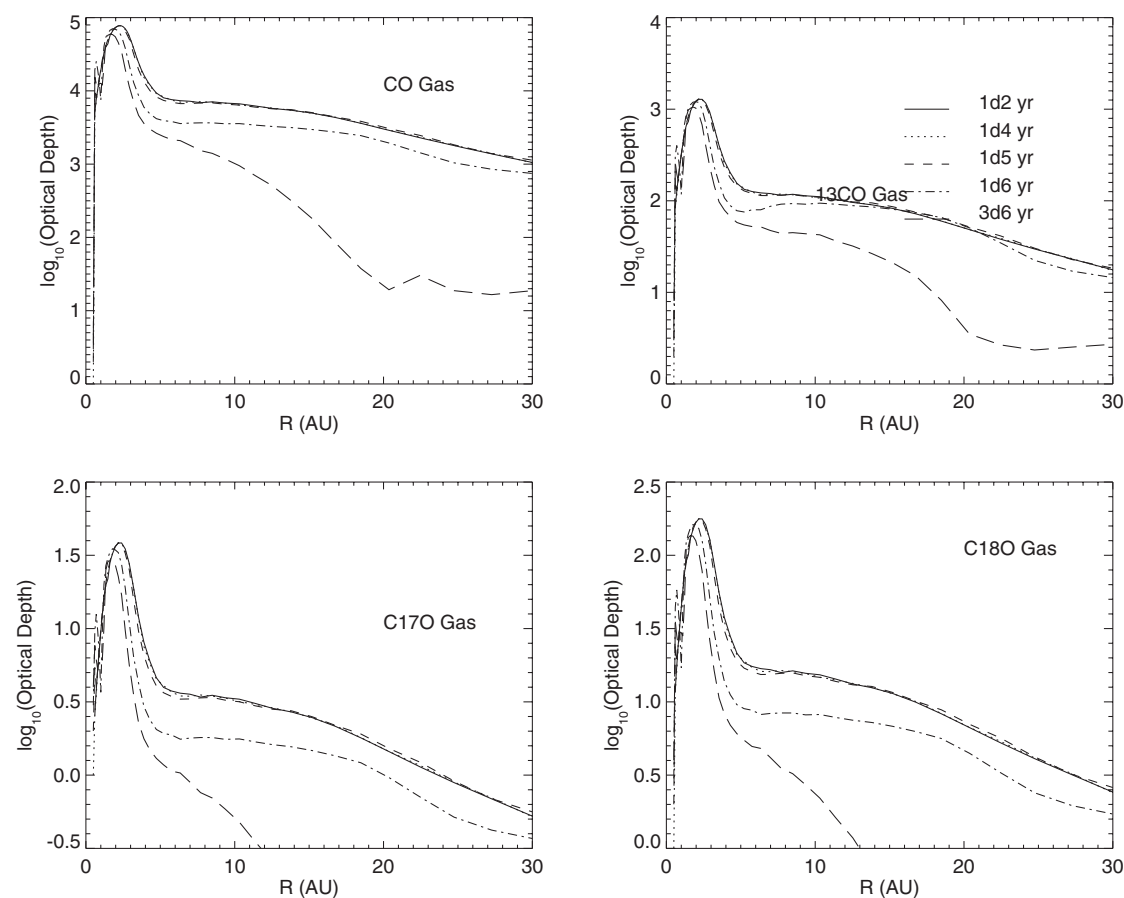

Figure 1. Vertical optical depth at $\mathrm{J}=3 \rightarrow 2$ of $\mathrm{CO},{ }^{13} \mathrm{CO}, \mathrm{C}^{17} \mathrm{O}$, and $\mathrm{C}^{18} \mathrm{O}$ at different time snapshots in the disk evolution. The depletion of all isotopologues of $\mathrm{CO}$ at late times is due to the formation of hydrocarbons. $\mathrm{C}^{17} \mathrm{O}$ and $\mathrm{C}^{18} \mathrm{O}$ probe the disk midplane in the giant planet-forming region of the disk. The model disk has mass $0.03 M_{\odot}$ spread over $70 \mathrm{AU}$.

Here we have verified that rare CO isotopologues are good probes of planet-forming protostellar disk midplanes. Such observations are good complements to the chemical imaging techniques of, for example, Qi et al. (2013), who search for CO freezeout by locating the inner edge of emission from molecules that are destroyed by $\mathrm{CO}$ gas, such as $\mathrm{H}_{2} \mathrm{CO}$ and $\mathrm{N}_{2} \mathrm{H}^{+}$.

This work was funded by NASA grant NNX10AH28G to SDR and KW. KW's work was conducted at the Jet Propulsion Laboratory, California Institute of Technology, under contract with the NASA Aeronautics and Space Administration.

\section{References}

Dodson-Robinson, S. E., Willacy, K., Bodenheimer, P., Turner, N. J., \& Beichman, C. A. 2009, Icarus, 200, 672

Landry, R., Dodson-Robinson, S. E., Turner, N. J., \& Abram, G. 2013, ApJ, 771, 80

Qi, C., Öberg, K. I., \& Wilner, D. J. 2013, ApJ, 765, 34 